\title{
ON THE BOUNDEDNESS OF AUTOMORPHIC FORMS
}

\author{
DAVID DRASIN AND C. J. EARLE ${ }^{1}$
}

1. Introduction. Let $\Gamma$ be a Fuchsian group acting on the unit disk $\Delta=\{z \in C:|z|<1\}$, and let $q \geqq 2$ be a fixed integer. The holomorphic function $\phi$ on $\Delta$ is called an automorphic form of weight $(-2 q)$ if

$$
\phi(A z) A^{\prime}(z)^{q}=\phi(z), \quad z \in \Delta, \quad A \in \Gamma .
$$

Let $\omega$ be a Poincaré normal polygon for $\Gamma$ in $\Delta$. With Bers [2], we call the automorphic form $\phi$ integrable if

$$
\|\phi\|=\iint_{\omega}|\phi(z)|\left(1-|z|^{2}\right)^{q-2} d x d y<\infty
$$

and bounded if

$$
\|\phi\|_{*}=\sup \left\{|\phi(z)|\left(1-|z|^{2}\right)^{q}: z \in \omega\right\}<\infty .
$$

$A_{q}(\Gamma)$ is the Banach space of integrable forms normed by (2), and $B_{q}(\Gamma)$ is the Banach space of bounded forms, normed by (3). If $\Gamma$ contains only the identity map, we write $A_{q}$ and $B_{q}$ for $A_{q}(\Gamma)$ and $B_{q}(\Gamma)$.

We shall prove

TheOREM 1. If $\Gamma$ is finitely generated, then $A_{q}(\Gamma) \subset B_{q}(\Gamma)$.

Theorem 2. If $A_{q}(\Gamma) \subset B_{q}(\Gamma)$, then the inclusion map is continuous.

Theorem 2 is a consequence of the closed graph theorem, and Theorem 1 is a consequence of this

Lemma. If $\Gamma$ is finitely generated and of the second kind, then there is an integrable form $\phi \in A_{q}(\Gamma)$ with the following properties:

(a) $\sup \{|\phi(z)|: z \in \omega\}<\infty$.

(b) If $\psi \in A_{q}(\Gamma)$, then $\psi(z)=f(z) \phi(z)$, where $f \in A_{q}$.

Since $\omega$ has finite (noneuclidean) area if and only if $\Gamma$ is a finitely generated group of the first kind [2], [6], our theorems are generalizations of the well-known fact that $A_{q}(\Gamma)=B_{q}(\Gamma)$ when $\omega$ has finite area.

2. Proof of the Lemma. It is well known [2], [6] that to each finitely generated Fuchsian group $\Gamma$ of the second kind there corresponds a compact bordered Riemann surface $\bar{X}$ with interior $X$ such that

Received by the editors June 2, 1967.

1 The second author was partially supported by NSF Grant GP 6145. 
$\Delta / \Gamma \subset X$, the set $X-\Delta / \Gamma$ is finite, and the natural map $\pi: \Delta \rightarrow \Delta / \Gamma$ is branched over only a finite number of points. Let $S=\left\{p_{1}, \cdots, p_{n}\right\}$ be the set of points $p \in X$ such that either $p \notin \Delta / \Gamma$ or the map $\pi$ is branched over $p$, and let $X^{\prime}=X-S$. For $p \in S$, we set $l(p)=\infty$ if $p \notin X$ and $l(p)=n$ if the map $\pi$ has a branch point of order $n-1$ over $p$.

If $1<l(p)<\infty$, then $p=\pi\left(z_{0}\right)$, where $z_{0} \in \Delta$ is the fixpoint of some $A \in \Gamma$ of order $n=l(p)$. A local parameter at $p$ is given by

$$
\zeta(z)=\left(z-z_{0}\right)^{n}\left(1-\bar{z}_{0} z\right)^{-n} .
$$

If $l(p)=\infty$, then there is a parabolic transformation $A \in \Gamma$ of the form

$$
(A z-a)^{-1}=(z-a)^{-1}+c, \quad a \in \partial \Delta,
$$

such that the function

$$
\zeta(z)=\exp (\pi i(z+a) / a c(z-a)), \quad z \in \Delta,
$$

for $z$ close to $a$, defines a local parameter in a punctured neighborhood of $p$.

Using these parameters at the points of $S$, one finds that every meromorphic differential of dimension $q$ on $X$ which lifts to a function $\phi \in A_{q}(\Gamma)$ is a multiple of the divisor

$$
D=-\sum_{p \in S}[q(1-1 / l(p))] p .
$$

Here $[x]$ denotes the greatest integer not exceeding $x$, with the understanding that $[q(1-1 / \infty)]=q-1$.

By Abel's theorem there is a meromorphic first order differential $\alpha$ on the double of $\bar{X}$ which is analytic and nonzero in $\bar{X}$. Furthermore, there is a function $g$ analytic in $X$ and continuous in $\bar{X}$ which has at each $p \in S$ a zero of order $[q(1-1 / l(p))]$ and no other zeros in $\bar{X}$. Then $\alpha^{q} / g$ is a meromorphic differential of dimension $q$ on $X$ with divisor $D$. This differential lifts to a holomorphic function $\phi$ on $\Delta$ which satisfies (1). It is easy to verify that $\phi$ is bounded in the fundamental polygon $\omega$. Hence $\|\phi\|<\infty$ and $\phi \in A_{q}(\Gamma)$.

On the other hand, each $\psi \in A_{q}(\Gamma)$ determines a meromorphic differential $\psi(z) d z^{q}$ on $X$ which is a multiple of the divisor $D$ of $\alpha^{q} / g$. This means that $\psi(z)=f(z) \phi(z)$, where $f(z)$ is the lift to $\Delta$ of an analytic function on $X$. (In particular, $f(A z)=f(z)$ for all $A$ in $\Gamma$.) We shall complete the proof of the Lemma by showing that $f \in A_{q}$.

To this end we choose a compact neighborhood $K \subset X$ of $S$, and we decompose $\Delta$ in to the $\Gamma$-invariant sets $\Delta_{1}=\pi^{-1}(K)$ and $\Delta_{2}=\Delta-\Delta_{1}$. It is clear that 


$$
\iint_{\Delta_{1}}|f(z)|\left(1-|z|^{2}\right)^{q-2} d x d y<\infty
$$

because the integrand is bounded in $\Delta_{1}$. On the other hand, if we put $\omega_{2}=\omega \cap \Delta_{2}$, then

$$
\sup \left\{\sum_{A \in \Gamma}\left|A^{\prime}(z)\right|^{q}: z \in \omega_{2}\right\}=M<\infty,
$$

and, since $\phi$ is continuous and nonzero in the closure of $\omega_{2}$,

$$
\inf \left\{|\phi(z)|: z \in \omega_{2}\right\}=\delta>0 .
$$

Thus,

$$
\begin{aligned}
\iint_{\Delta_{2}}|f(z)| & \left(1-|z|^{2}\right)^{q-2} d x d y \\
& =\sum_{A \in \Gamma} \iint_{A \omega_{2}}|f(z)|\left(1-|z|^{2}\right)^{q-2} d x d y \\
& =\sum_{A \in \Gamma} \iint_{\omega_{2}}|f(A \zeta)|\left(1-|A \zeta|^{2}\right)^{q-2}\left|A^{\prime}(\zeta)\right|^{2} d \xi d \eta \\
& =\sum_{A \in \Gamma} \iint_{\omega_{2}}|f(\zeta)|\left(1-|\zeta|^{2}\right)^{q-2}\left|A^{\prime}(\zeta)\right|{ }^{q} d \xi d \eta \\
& \leqq M \delta^{-1} \iint_{\omega_{2}}|f(\zeta) \phi(\zeta)|\left(1-|\zeta|^{2}\right)^{q-2} d \xi d \eta \leqq M \delta^{-1}\|\psi\| .
\end{aligned}
$$

Combining this inequality with (4), we find that $\|f\|<\infty$. The Lemma is proved.

3. Proof of Theorem 1. We may assume that $\Gamma$ is of the second kind, since otherwise the theorem is well known. By the Lemma, if $\psi \in A_{q}(\Gamma)$, then $\psi=f \phi$, with $f \in A_{q}$ and $\phi$ bounded in $\omega$. Choose $M$ so that $|\phi(z)| \leqq M, z \in \omega$. Then

$$
\|\psi\|_{*}=\sup \left\{|\psi(z)|\left(1-|z|^{2}\right)^{q}: z \in \omega\right\} \leqq M\|f\|_{*} .
$$

But $\|f\|_{*}$ is finite because $A_{q}$ is contained in $B_{q}$ by $[2$, p. 199]. The theorem is proved.

4. Proof of Theorem 2. By assumption, the injection $i: A_{q}(\Gamma)$ $\rightarrow B_{q}(\Gamma)$ is defined on all of $A_{q}(\Gamma)$. Since for each $z \in \Delta$ the map $\phi \rightarrow \phi(z)$ is continuous on both $A_{q}(\Gamma)$ and $B_{q}(\Gamma)$, the graph of $i$ is closed. Hence $i$ is a continuous map. 
5. Remarks. (i) Let $\Gamma$ be finitely generated and of the second kind. Let $E_{q}$ be the Banach subspace of $A_{q}$ consisting of those $f$ such that $f(A z)=f(z)$ for all $A \in \Gamma$. By the Lemma, the map $f \rightarrow f \phi$ is a continuous bijective map from $E_{q}$ to $A_{q}(\Gamma)$. Hence, by the open mapping theorem it is an isomorphism.

(ii) We are unable to prove that $A_{q}(\Gamma) \subset B_{q}(\Gamma)$ for arbitrary $\Gamma$. However, it is true that $A_{q}(\Gamma) \cap B_{q}(\Gamma)$ is always dense in $A_{q}(\Gamma)$. (This observation has been made independently by L. Bers.) To prove this, we note first that polynomials are dense in $A_{q}$, as an immediate consequence of [3, Lemma 1]. But a theorem of Bers [2], [4] asserts that the Poincaré series

$$
\Theta \Phi(z)=\sum_{A \in \Gamma} \Phi(A z) A^{\prime}(z)^{q}
$$

defines a continuous map of $A_{q}$ onto $A_{q}(\Gamma)$. Hence the functions $\Theta P, P$ a polynomial, are dense in $A_{q}(\Gamma)$. Finally, each such function belongs to $B_{q}(\Gamma)$ by Godement's theorem on the boundedness of Poincaré series [5, Theorem 5 bis]; [1].

\section{REFERENCES}

1. L. V. Ahlfors, Eine Bemerkung über Fuchssche Gruppen, Math. Z. 84 (1964), $244-245$.

2. L. Bers, Automorphic forms and Poincaré series for infinitely generated Fuchsian groups, Amer. J. Math. 87 (1965), 196-214.

3. - A non-standard integral equation with applications to quasiconformal mappings, Acta Math. 116 (1966), 113-134.

4. C. J. Earle, A reproducing formula for integrable automorphic forms, Amer. J. Math. 88 (1966), 867-870.

5. R. Godement, Séries de Poincaré et Spitzen-formen, Séminaire Henri Cartan, 10e année (1957/1958), Exposé 10.

6. A. Marden, On finitely generated Fuchsian groups, Comment. Math. Helv. 42 (1967), 81-85.

Purdue University and

Cornell University 